\title{
Benign cystic peritoneal mesothelioma
}

\author{
D S Bhandarkar, V J Smith, D A Evans, T V Taylor
}

\begin{abstract}
The well defined but rare entity of benign cystic mesothelioma is reported. The aetiology of this neoplasm remains obscure. The presenting features make a precise preoperative diagnosis difficult; information provided by computed tomography and cytology may help. A firm diagnosis can only come from electron microscopic or immunohistochemical examination of the tumour. Diagnostic accuracy and diligent follow up are essential because, although the tumour is considered benign, it does tend towards local recurrence.
\end{abstract}

(F Clin Pathol 1993;46:867-868)

Department of

Surgery, Manchester

Royal Infirmary,

Oxford Road,

Manchester M13 9WL

D S Bhandarkar

D A Evans

T V Taylor

Department of

Histopathology

V J Smith

Correspondence to:

Mr T V Taylor

Accepted for publication

17 March 1993
Benign cystic peritoneal mesothelioma (BCPM) is a neoplasm composed of multiple small fluid-filled cysts. In terms of malignant potential it lies somewhere between two other neoplasms of mesothelial origin: adenomatoid tumour and malignant peritoneal mesothelioma. ${ }^{1}$ The more common peritoneal adenomatoid tumour is usually an incidental finding at laparotomy and rarely causes symptoms. In contrast, the malignant mesothelioma, which is known to develop after exposure to asbestos, frequently metastasises

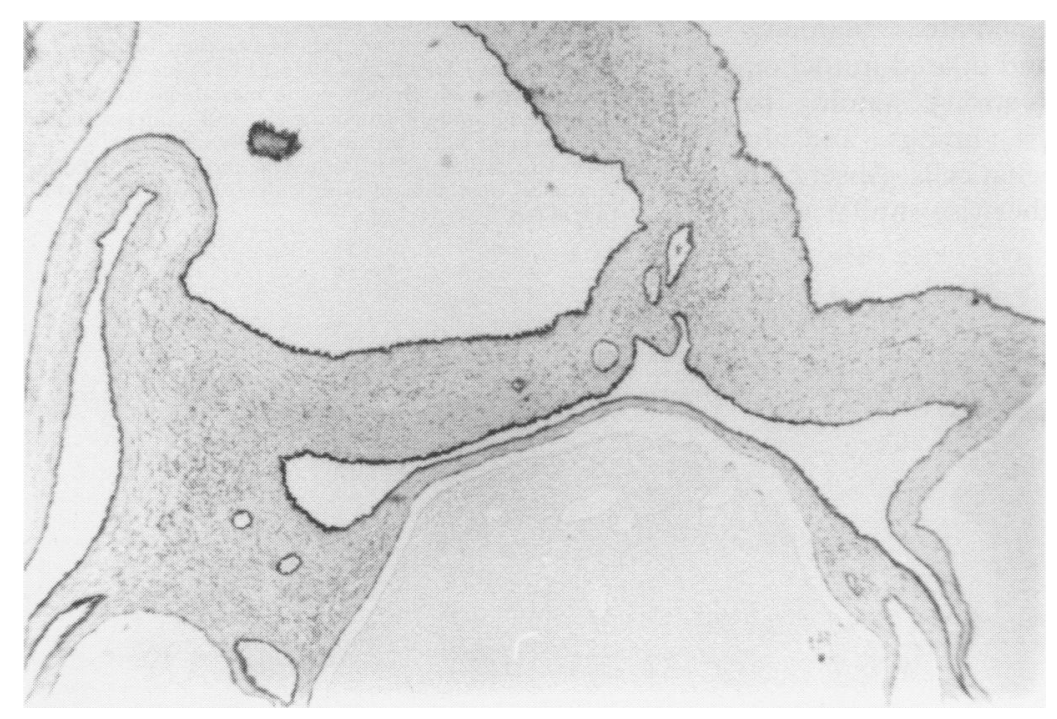

Cyst lining stained with anti-cytokeratin antibody. Note the intense staining of the lining cells (immunoperoxidase for CAM 5.2). and is fatal. BCPM does not metastasise and therefore is not truly malignant, but frequently recurs locally. BCPM is more common in women of reproductive age; there have been only 18 cases reported in men. ${ }^{2}$

\section{Case report}

A 47 year old man presented with intermittent, generalised abdominal pain of three years duration. Three months before admission he had noticed a swelling on the right side of his abdomen which had grown steadily in size. There were no other symptoms. On examination there was a large, lobulated mass with ill defined margins occupying the entire right side of the abdomen and extending into the hypogastrium. A smaller mass with similar features was palpable in the left iliac fossa. There was no hepatosplenomegaly or ascites. Serum biochemistry was normal. Ultrasonography showed a large, intraperitoneal, multicystic mass not obviously arising from any solid organ. Ultrasonographically guided needle aspiration yielded pale yellow fluid which showed mesothelial cells on cytology. At laparotomy the multicystic mass was found to occupy almost the entire abdomen and was adherent to small bowel, large bowel, liver and bladder. The tumour was resected except where inseparable from viscera. The patient made an uneventful postoperative recovery and no further treatment is planned at the time of writing. Three months after surgery the patient remained asymptomatic.

Grossly, the specimen was a gelatinous mass. Its cut surface showed multiple cystic spaces. Microscopically, the tissue showed cystic spaces of varying sizes separated by fibrous septa. The spaces were lined by a single layer of flattened or cuboidal cells. On immunohistochemical staining, these cells stained postively for cytokeratin (CAM 5.2) and negatively for factor VIII related antigen, thereby confirming their mesothelial origin (figure). The histological diagnosis was of benign cystic peritoneal mesothelioma.

\section{Discussion}

The clinical and pathological findings described in relation to our case represent typical features of BCPM.

In 1889 Henke $^{3}$ reported a case of "multiple cystic lymphangiomalike tumour". Over the next hundred years various similar 
growths were described and it was only in 1980 that the now commonly accepted term, "benign cystic mesothelioma", was used. ${ }^{4}$ The mesothelial origin of such growths had been demonstrated by Mennmeyer and Smith in $1979 . .^{5}$ The aetiology remains obscure. In particular there is no association with asbestos exposure, previous abdominal surgery, or abdominal trauma. ${ }^{1}$

Abdominal pain, tenderness, and distension, usually in association with a pelvic or abdominal mass, are the common presenting features. Ultrasonography can give useful information but computed tomography scanning is the investigation of choice. With this imaging modality the differential diagnoses include peritoneal lymphangioma, pseudomyxoma peritonei, and cystadenoma or cystadenocarcinoma of the ovary.

Aspiration cytology can be useful in making a preoperative diagnosis. ${ }^{3}$ Typically, the aspirate from a BCPM contains mesothelial cells showing focal presence of a brush border. Conditions such as cirrhosis, viral infections, and connective tissue disorders with mesothelial hyperplasia can also yield an aspirate with mesothelial cells, but in these conditions the cells tend to form clusters and aggregates as opposed to the large flat sheets seen in BCPM.

At surgery the tumour typically consists of multiple, translucent cysts forming a confluent mass. The tumour has been reported to include various parts of the serosa of the bowel and sometimes spreads to liver, spleen, and pancreas. Rarely the tumour may present as free floating pelvic cysts. ${ }^{6}$ Histopathologically, BCPM is composed of multiple mesothelial-lined cystic spaces separated by a delicate fibromuscular stroma. Mild to moderate inflammatory changes are occasionally seen. The mesothelial origin is confirmed by electron microscopy or immunohistochemistry. The former shows the characteristics of mesothelial cells-slender microvilli on the luminal surface of the cells, desmosomes, intracytoplasmic intermediate filaments, endoplasmic reticulum and dilated mitochondria. The latter shows strong staining for cytokeratin in the cyst linings and for vimentin in the subepithelial cells. Absence of both these features in otherwise similar tissue suggests the diagnosis of cystic lymphangioma. ${ }^{7}$ Special stains (Masson Trichrome) can be used to demonstrate the presence of muscle fibres in the cyst lining of cystic lymphangioma. These muscle fibres are absent from the cyst walls of BCPM. Adenomatoid mesothelial tumours and the malignant mesothelioma can be easily differentiated from BCPM by their gross and histological characteristics. Accurate diagnosis of BCPM is important because, though benign, these tumours tend to recur locally.

BCPM is treated by surgical resection. When complete removal is impossible due to adherence, marsupialisation of the remaining cysts is recommended. Radical resection is not advocated if this puts vital structures at risk. Intraperitoneal tetracycline has been used to sclerose residual tumour after surgical resection ${ }^{8}$ but there is no evidence that this is beneficial. Chemotherapy and radiotherapy are of no value. Considering the potential of this tumour to recur, close follow up is essential. Substantial recurrence usually warrants further resection.

The prognosis of BCPM is excellent. The reported recurrence rate is slightly higher in women $(40-50 \%)$ than in men $(33 \%){ }^{2}$ Surprisingly, there has only been one reported death from BCPM, the patient dying as a result of local tumour effects 12 years after refusing surgery.

DSB's training in the United Kingdom is supported by a grant from Rameshwardas Birla Smarak Kosh, Bombay, India.

1 Nirodi NS, Cowry DS, Wallace RJ, et al. Cystic mesothelioma of the pelvic peritoneum. Two case reports. $\mathrm{Br} \mathcal{F}$ Obstet Gynaecol 1989;91:201-4.

2 Canty MD, Williams J, Volpe RJ, et al. Benign cystic mesothelioma in a male. Am $\mathcal{F}$ Gastroenterol 1990;85: 311-15.

3 Baddoura FK, Varma VA. Cytology of multicystic peritoneal mesothelioma. Acta Cytol 1990;34:524-8.

4 Moore JH, Crum CP, Chandler JG, et al. Benign cystic mesothelioma. Cancer 1980;45:2395-9.

5 Mennemeyer $R$ Smith $M$. Multicystic peritoneal mesothelioma: a report with electron microscopy of a case mimicking intraabdominal cystic hygroma (lymphangioma). Cancer 1979;44:692-8.

6 Weiss SW, Tarssoli FA. Multicystic mesothelioma. An analysis of pathologic findings and biologic behaviour in 37 cases. Am $\mathcal{F}$ Surg Pathol 1988;12:737-46.

7 Raafat F, Egan M. Benign cystic mesothelioma of peritoneum. Immunohistochemical and ultrastructural features in a child. Paediatr Pathol 1988;8:321-9.

8 Benson RL, Williams JH. Peritoneal cystic mesothelioma: successful treatment of a difficult disease. $\mathcal{F}$ Urol 1990; 143:347-8. 